\title{
Weak Communication in Radio Networks *
}

\author{
Tomasz Jurdziński*†, Mirosław Kutyłowski†, and Jan Zatopiański† \\ * Institute of Computer Science, Technical University of Chemnitz, Germany \\ $\dagger$ Institute of Computer Science, Wrocław University, Poland \\ \#Institute of Mathematics, Wrocław University of Technology and Dept. of Math. and Computer \\ Science, A. Mickiewicz University, Poznań, Poland
}

\begin{abstract}
Quite often algorithms designed for no-collision-detection radio networks use a hidden form of collision detection: it is assumed that a station can simultaneously send and listen. Then, if it cannot hear its own message, then apparently a collision has occurred. IEEE Standard 802.11 says that a station can either send or listen to a radio channel, but not both. So we consider a weak radio network model with no collision detection where a station can either send or receive signals. Otherwise we talk about strong model.

We show that power of weak and strong radio networks differ substantially in deterministic case. On the other hand, we present an efficient simulation of strong by weak ones, with randomized preprocessing of $O(n)$ steps and $O(\log \log n)$ energy cost.
\end{abstract}

\section{Introduction}

Radio networks became today an attractive alternative for wired ones. Luck of a fixed infrastructure and mobility allow to create self-adjusting network of independent devices that is useful for application areas such as disaster-relief, law-enforcement, and so on. The main advantages are dynamic topology and simple communication protocol. However, colliding messages create many challenging problems.

We are interested in networks of stations communicating via radio channels. The stations are hand-held bulk produced devices running on batteries. They have no ID's or serial numbers, some of the stations are switched off, but somehow the network has to organize itself, despite the lack of a central control. It is often overlooked by designers of algorithms for radio networks that many current technologies do not provide capability of listening while a station is sending (IEEE Standard 802.11). On the other hand, simultaneous sending and listening for collisions is a feature used in many algorithms. Our goal is to check how does it influence problem complexity on radio networks.

Model A radio network (RN, for short) consists of several processing units called stations. A RN is synchronized by a global clock (for instance based on GPS). Communication is possible in time slots, called here steps. We assume that there is a single communication channel available to all stations (so we consider single-channel, singlehop RN's). If during a step stations may either send (broadcast) a message or listen to

\footnotetext{
* the first author was supported by DFG grant GO 493/1-1, the second author by KBN grant 7
} T11C 032 21, and the third author by KBN grant 7 T11C 03220. 
the channel, then we talk about weak RN. If both operations can be performed by a station, then we have to do with the strong RN. (In the literature, by a RN the authors usually mean the strong RN.) If exactly one station sends, then all stations that listen at this moment receive the message sent. We assume in this paper that if at least two stations send, then a collision occurs and the stations that listen do not receive messages and even cannot recognize that messages have been sent (no collision-detection $R N$ ). If a station is the only station that sends a message during step $i$, then we say that the station succeeds and that step $i$ is successful.

The stations of a RN fall into two categories. Some of them are switched off and do not participate in the protocol. The other stations are active and we assume that they stay active during the whole protocol. The set of all active stations is called active set. During a step an active station might be either awake or asleep. The first case occurs when a station sends a message or listens to the communication channel.

Complexity measures There are two main complexity measures for $\mathrm{RN}$ algorithms: time complexity and energy cost [?]. Time complexity is the number of steps executed. Energy cost is the maximum over all stations of the number of steps in which the station is awake. Low energy cost is a desirable feature: if a station which is awake for a longer time, it may fail to operate due to batteries exhaustion [?]. So a station which is awake much longer than the other stations is likely to fail and break down the protocol.

For further discussion on the RN model and its complexity measures, see e.g. [?,?,?,?,?,?,?,?].

Fundamental tasks Due to the lack of ID numbers, uncertainty about the number of active stations, switching off the stations, and so on, certain problems become hard for RN's, while they are much easier in the wired networks. Some of such basic tasks are the following:

Leader election: active stations have to choose a single station, called leader, among themselves. That is, at the end of the protocol a single station has status leader and all other active stations have status non-leader.

Initialization: consecutive ID numbers are to be assigned to active stations with no ID's.

Renumeration: consecutive ID numbers are to be assigned to active stations having some unique ID's, but in the range bigger than the number of stations.

\section{Previous Results}

Due to the role of energy cost, there is a lot of interest for algorithms that are time and energy efficient. However, research has been focused on the strong model.

For leader election, the first energy efficient algorithm has been designed in [?]. The authors present a randomized algorithm that for $n$ stations elects a leader ( $n$ must be known to the stations) in time $O(\log f)$ and energy $O\left(\log \log f+\frac{\log f}{\log n}\right)$ with probability $1-1 / f$ for any $f \geq 1$. Moreover, they get algorithms that elect a leader within $O(\log n)$ energy cost and $O\left(\log ^{2} n\right)$ time with probability $1-\frac{1}{n}$ if $n$ is unknown. [?] presents an algorithm which achieves $O(\log n)$ time and $O\left(\log ^{*} n\right)$ energy cost with 
probability $1-\frac{1}{n}$ when $n$ is known to the stations. If $n$ is unknown, a general algorithm that approximates the value of $n$ within a constant multiplicative factor can be applied [?]. It uses time $O\left(\log ^{2+\varepsilon} n\right)$ and energy $\operatorname{cost} O\left((\log \log n)^{\varepsilon}\right)$ with probability $1-\frac{1}{n}$.

It is impossible to elect a leader deterministically when stations are indistinguishable, due to symmetry. So we apply deterministic algorithms only when the stations have unique ID's, say in the range [1..n] (but any subset of these ID's may correspond to active stations). A simple leader election deterministic algorithm for this case is given in [?,?]. Its energy cost is $\log n+O(1)$, run time is $n+O(1)$. A recursive procedure that works in time $O(n)$ and energy $O\left(\log ^{\varepsilon} n\right)$ is given in [?].

An energy efficient solution to initialization problem is proposed in [?]: with probability at least $1-\frac{1}{n}$ its energy cost is $O(\log \log n)$ and execution time is $O(n)$. However, the number of stations $n$ must be known beforehand. The algorithm can be easily generalized to the case when an approximation of $n$ within a constant multiplicative factor is known. Hence using size-approximation algorithm from [?], the same time and energy efficiency can be obtained for an unknown $n$.

\section{New Results}

The main result of this paper is a randomized protocol for simulating algorithms for strong RN's by weak RN's.

Theorem 1. An algorithm for strong $R N$ with run time $T$ and energy cost $E$ can be simulated by a randomized weak $R N$ with run time $O(T)$ and energy cost $O(\max (E, T / n))$. A preprocessing is required with time $O(n)$ and energy cost $O(\log \log n)$.

Before we construct the simulation algorithm (presented in Sections ??-??), we show in Section ?? that the situation is much different in deterministic setting:

Theorem 2. Leader election has energy $\operatorname{cost} \Omega(\log n)$ on deterministic weak RN's.

This result is interesting since on the other hand there is a solution for the strong model that works in time $O(n)$ and energy $O\left(\log ^{\varepsilon} n\right)$ [?]. For a more practical solution with energy $\operatorname{cost} O(\sqrt{\log n})$ see [?].

\section{Gap between Weak and Strong Models}

In this section we prove Theorem ??. Let the stations have distinct identifiers in the range $\{1, \ldots, n\}$, but the number of active stations might be arbitrary up to $n$. We construct sets $A_{0}, A_{1}, \ldots$ such that for every $i$, if $I \subseteq A_{i}$ is the active set, $j \in I$ and $\left|A_{i}\right|>1$, then the station $j$ cannot decide if it is the leader after step $i$. Moreover, we impose some other properties that guarantee that $\left|A_{i}\right|>0$ and the equality $A_{i}=\{j\}$ implies that station $j$ is awake at least $\Omega(\log n)$ times up to step $i$ (if the active set is equal to $A_{i}$ ).

Let $A_{0}=\{1, \ldots, n\}$. For $i>0$ let $S_{i}\left(R_{i}\right.$ resp.) be a set of stations that send a message (listen, resp.) during step $i$, if the active set is $A_{i-1}$. For definition of $A_{i}$ we use an auxiliary ,weight" $w_{i}(j)$ of station $j$ at step $i$. It fulfills the following invariant: $\sum_{j \in A_{i}} w_{i}(j)=n$ for $i \in \mathbb{N}$. We start with $w_{0}(j)=1$ for $i=1, \ldots, n$. We put $A_{i}=A_{i-1} \backslash R_{i}$ if $\sum_{j \in S_{i}} w_{i}(j)>\sum_{j \in R_{i}} w_{i}(j)$ and $A_{i}=A_{i-1} \backslash S_{i}$ otherwise. If $A_{i}=A_{i-1} \backslash R_{i}$, then 
- $w_{i}(j)=w_{i-1}(j) \quad$ for every $j \in A_{i} \backslash S_{i}$

- $w_{i}(j)=w_{i-1}(j)+v_{j} \quad$ for every $j \in S_{i}$, where $v_{j}=\frac{w_{i-1}(j)}{\sum l \in S_{i} w_{i-1}(l)} \cdot \sum_{l \in R_{i}} w_{i-1}(l)$.

If $A_{i}=A_{i-1} \backslash S_{i}$, then the weights are defined similarly. Obviously, the weights defined in such a way satisfy the invariant. Note that above rules guarantee that $w_{i}(j) \leq 2 w_{i-1}(j)$ for every $i, j$. Moreover, $w_{i}(j)>w_{i-1}(j)$ if and only if $j$ is awake at the step $i$ (for active sets contained in $A_{i}$ ).

Proposition 1. Let $j \in A_{i}$. Then station $j$ does not hear any message up to step $i$ for any active set $I \subseteq A_{i}$ such that $j \in I$.

Proof. The proof is by induction on $i$. The case $i=0$ is obvious. Assume that the property holds for $i-1$. If $A_{i}=A_{i-1} \backslash R_{i}$, then all awake stations from $A_{i}$ are sending and nobody listens. So no information is reaching anybody. If $A_{i}=A_{i-1} \backslash S_{i}$, then all awake stations are listening, but nobody sends a message at step $i$, so no awake station of $A_{i}$ gets any information.

$\square$ Proposition ??

Proposition 2. If the active set is equal to $I \subseteq A_{i}$ and $j \in I$, then $w_{i}(j) \leq 2^{l}$, where $l$ is the number of steps among steps 1 through $i$ during which station $j$ was awake.

Proof. We show it by induction with respect to $i$. The case $i=0$ is obvious. For $i>0$, it is enough to observe that the new factor added to the weight is smaller than the „old" weight and the weight may increase only when a station is awake. $\square$ Proposition ??

By Proposition ??, if $\left|A_{i}\right|>1$ and $j \in A_{i}$, then the station $j$ is unable to distinguish after $i$ steps between active sets $\{j\}$ and $A_{i}$. Let $l$ be the leader elected for active set $A_{i}$ and $j \in A_{i} \backslash\{l\}$. Then station $j$ cannot decide if it is the leader after the step $i$ (it becomes the leader if the active set is equal to $\{j\}$ and it is not the leader if the active set is $A_{i}$ ). We conclude that if $\left|A_{i}\right|>1$, then the algorithm cannot terminate after step $i$. Since $\left|A_{i}\right|>0$ for every $i>0$, the algorithm can terminate after step $i$ for the active set $A_{i}$ if and only if $\left|A_{i}\right|=1$. Let $j$ be the only element of such $A_{i}$. Then $w_{i}(j)=n$ and by Proposition ??, station $j$ is awake at least $\log n$ times during steps $1, \ldots, i$.

\section{Algorithmic Tricks for Weak RN's}

Although energy cost of deterministic leader election is different for weak and strong RN's, in the randomized case the best algorithms can be run on weak RN's [?], using tricks presented below. Moreover, a linear approximation of the number of active stations may be obtained efficiently by randomized algorithm, using a method designed for the strong model. This indicates that randomization may really help. In this section we make some observations that help to design "strong algorithms" in weak RN's.

Previous RN algorithms use very often the following basic trick ([?,?,?,?]): given $n$ active stations, each of them sends with probability $1 / n$. Then with probability $n \cdot \frac{1}{n}$. $\left(1-\frac{1}{n}\right)^{n-1} \approx \frac{1}{e}$ exactly one station sends. In the strong model, the station that succeeds may recognize it. This experiment can be used for randomized leader election in the strong model: we apply the experiment $\ln n$ times and use Basic Algorithm for leader election over those stations that have been successful during $\ln n$ experiments (with ID's equal to numbers of successive steps). 
Pairing trick in the weak model The method described above cannot be implemented directly in the weak model. However, this problem can be eluded as follows:

1. each station chooses uniformly at random to be either sender or receiver,

2. each sender transmits a message with probability $\frac{2}{n}$,

3. each receiver listens with probability $\frac{2}{n}$ and if a message is received, it responds with the same message,

4. each sender that have sent a message at step 2 listens. If it receives its own message now, then it was the only station sending at step 2 .

In this procedure it may happen that a station sending a message is successful, but it does not receive a confirmation since more than one receiver is sending it. Nevertheless, we show that the probability that one station sends a message and receives a confirmation is bounded from below by a constant close to $\frac{1}{e^{2}}$. First observe that the probability that there are more than $n / 2+n^{2 / 3}$ senders is at most $e^{n^{1 / 3} / 6}$ by Chernoff bound [?]. If there are $\frac{n}{2}+d$ senders, $d<n^{2 / 3}$, then probability of getting a confirmation equals

$$
\begin{array}{r}
\left(\left(\frac{n}{2}+d\right) \cdot \frac{2}{n} \cdot\left(1-\frac{2}{n}\right)^{n / 2-1+d}\right) \cdot\left(\left(\frac{n}{2}-d\right) \cdot \frac{2}{n} \cdot\left(1-\frac{2}{n}\right)^{n / 2-1-d}\right) \\
\approx \frac{1}{e^{2}} \cdot\left(1-\frac{d^{2}}{(n / 2)^{2}}\right)>\frac{1}{e^{2}} \cdot\left(1-\frac{4}{n^{2 / 3}}\right)
\end{array}
$$

Partial initialization Assume that among $n$ stations we have already a fraction $n / d$ stations that have assigned unique ID numbers in the range $1 . . n / d$ - we call them the first group. Then we are able to run an algorithm for the strong model on the remaining $n-n / d$ stations, called the second group, in the following way: step $i$ of the algorithm is simulated by the steps $2 i-1$ and $2 i$. At step $2 i-1$ the processors of the second group execute step $i$ of the algorithm. Additionally, the station $1+i \bmod \frac{n}{d}$ from the first group listens. At step $2 i$ it sends the message received (if any). In turn, all stations of the second group that have been sending during step $2 i-1$ are listening. This enables them to recognize a collision, if it occurred at step $2 i-1$.

Observe that for simulating $n$ steps of an algorithm for strong model each of the stations of the first group is awake for $2 d$ steps. If $d$ is small, for instance $\log \log n$, then this energy cost might be smaller than the energy cost occurring for the stations of the second group, and therefore acceptable.

\section{Initialization}

The best known algorithm for initialization [?] works in the strong model only: its key point is that the stations assign themselves random ID's in the range $1, \ldots, m$ for some $m \in \mathbb{N}$ and verify their uniqueness in such a way that stations with ID equal to $j$ send at step $j$ for $j=1, \ldots, m$ and check for collisions. For the weak model one may try to re-use the pairing trick, i.e. split stations at random into senders and receivers, then each sender sends a message and each receiver listens in the step equal to picked number and they confirm uniqueness as in the pairing trick. However, it does not work well if there are few stations choosing ID's from a big set - but just this situation leads to double logarithmic energy cost in [?].

Let us outline our initialization algorithm. It consists of three main phases: 
1. Assign temporary unique ID's from the set $\left\{1, \ldots, c_{1} n\right\}$ to a subset $U$ of stations such that $|U| \geq c_{2} n$, for some constants $c_{1}$ and $c_{2}$.

2. Run ,renumeration” procedure on the elements of $U$. This procedure assigns distinct consecutive ID's, starting with 1 , to a large subset of $U$, say $I$.

3. Using the partial initialization trick run the initialization algorithm from [?] on the stations that do not belong to $I$ (with $I$ as the first group).

Phase 1 Let us describe in detail the first phase:

(A) Split the set of stations into subsets $S_{0}$ and $S_{1}$. Each station chooses bit $b$ uniformly at random. The stations that set $b=i$ belong to the set $S_{i}$.

(B) Each station picks an integer from the set $\{1, \ldots, 3 n\}$ uniformly at random. Let $S_{i}^{j}$ be the set of elements of $S_{i}$ which choose number $j$.

(C) for $j \leftarrow 1$ to $n$ do the stations from $S_{1}^{j}$ and $S_{0}^{j}$ verify if $\left|S_{1}^{j}\right|=\left|S_{0}^{j}\right|=1$ : first all elements of $S_{1}^{j}$ send and all elements of $S_{0}^{j}$ listen; in the next step all elements of $S_{1}^{j}$ listen and each element of $S_{0}^{j}$ sends the message just heard; if the message comes through, then $\left|S_{1}^{j}\right|=\left|S_{0}^{j}\right|=1$ and the only element of $S_{1}^{j}$ sets ID $\leftarrow j$.

Lemma 1. With probability at least $1-\frac{1}{n^{2}}$ the number of stations with assigned ID's is bigger than cn, for a constant $c$ independent of $n$.

Proof. As we have already seen $n / 2-n^{2 / 3} \leq\left|S_{b}\right| \leq n / 2+n^{2 / 3}$ for $b=0,1$ with probability $1-e^{-n^{1 / 3} / 6}$. Thus assume that $\left|S_{i}\right|=c_{i} n$, for $i=0,1$, where $c_{0}, c_{1}$ are very close to $1 / 2$, and $c_{0}+c_{1}=1$. Let us consider the number of sets $S_{b}^{j}$ such that $\left|S_{b}^{j}\right|=1$. We call the stations from such $S_{b}^{j}$,s successful. Imagine that we pick numbers for stations sequentially. If the $j$ th station of $S_{b}$ picks a number chosen already by the other station, then we say that a collision occurs. Let $p_{j}$ be the probability that a collision occurs in the $j$ th step. It depends very much on the previous choices, but every time $p_{j} \leq \frac{j-1}{3 n}$. Let $X$ be the number of collisions. We can bound (from above) the probability that $X>\alpha$ (for any $\alpha$ ) by the probability that $Y=\sum_{i=1}^{c_{b} n} Y_{i}>\alpha$, where $Y_{i}$ are independent random ,0-1" variables such that $\mathbf{P}\left[Y_{j}=1\right]=\frac{j}{3 n}$. Observe that $\mathbf{E}[Y]=\sum_{j=1}^{c_{b} n} \frac{j}{3 n}=\frac{c_{b} n\left(c_{b} n-1\right)}{2 \cdot 3 n}$. Together with Chernoff Bound [?] this yields

$$
\mathbf{P}\left[X>\frac{\left(c_{b} n\right)^{2}}{3 n}\right] \leq \mathbf{P}\left[Y>\frac{\left(c_{b} n\right)^{2}}{3 n}\right] \leq e^{-\frac{c_{b} n\left(c_{b} n-1\right)}{2 \cdot 3 \cdot 3 n}}=O\left(n^{-4}\right)
$$

for $n$ large enough. A collision may prevent at most two stations from being successful. Thus the number of stations that are not successful is at most $2 X$. So the number of successful stations in $S_{b}$ is at least $c_{b} n-2 X \geq c_{b}-\frac{c_{b}^{2} n^{2}}{3 n}=n\left(c_{b}-\frac{c_{b}^{2}}{3}\right) \geq c_{b}^{\prime} n$ with high probability, where $c_{b}^{\prime}$ is a constant very close to $\frac{5}{12}$.

Now let us assume that the set of successful stations in $S_{b}$ (for $b=0,1$ ) consists of $d_{b} n$ elements, where $d_{b} \geq \frac{1}{3}$. Fix $d_{0} n$ numbers associated to successful stations from $S_{0}$ (each subset of $\{1, \ldots, 3 n\}$ of size $d_{0} n$ has equal probability). Now imagine that we assign numbers to successful stations from $S_{1}$ sequentially. The probability that the $(j+1)$ st station is , paired" with a successful station from $S_{0}$ is not smaller than $\frac{d_{0} n-j}{3 n-j} \geq$ 
$\frac{d_{0} n-j}{3 n}$. The expected number of ,,successful pairs" is not smaller than $\sum_{j=1}^{d} \frac{d_{0} n-j}{3 n} \geq$ $\frac{1}{3 n} \cdot \frac{d n(d n-1)}{2} \geq d^{\prime} n$, where $d=\min \left(d_{0}, d_{1}\right)$ and $d^{\prime}$ is a constant. Finally, by Chernoff Bound [?], the number of ,,successful pairs” is bigger than $d^{\prime} n / 2$ with high probability, assuming that the sizes of $S_{0}$ and $S_{1}$ are close each other and the number of successful stations in them are bigger than $n / 3$. But this assumptions are satisfied with high probability, too.

Renumeration Now, we recall the following result from [?]: For no-collision detection $\mathrm{RN}$ there is a deterministic leader election algorithm with $\log ^{*} n$ energy cost and time $O(n)$ that works for an arbitrary set of stations with distinct identifiers in the range 1..n such that the number of active stations is at least $c \cdot n$ for any fixed constant $c>0$. In fact this algorithm works in the weak model. Moreover, it consists of $O\left(\log ^{*} n\right)$ phases and the following properties are satisfied in the phase $i$ :

- At least $c_{i} x_{i}$ active stations called masters participate in the phase.

- A set of at least $\frac{c}{2} \cdot s_{i}$ active stations called slaves is assigned to each master. The slaves of the master are numbered by consecutive numbers $1,2, \ldots$

- Only one master remains after the last phase.

We have $x_{1}=n, s_{1}=2 / c, c_{1}=c$ and $c_{i+1}=c_{i} / 2, x_{i+1}=x_{i} / 2^{s_{i}}, s_{i+1}=c_{i+1} \cdot s_{i} \cdot 2^{s_{i}}$ for $i>1$. So, the number of masters and their slaves at phase $i$ is not smaller than $\frac{c}{2} \cdot s_{i} c_{i} x_{i}$. Observe that $c_{i}=\frac{c}{2^{i-1}}, \frac{c}{2} \cdot c_{1} x_{1} s_{1}=c n$, and $c_{i+1} x_{i+1} s_{i+1}=\frac{c_{i}}{2} \cdot \frac{x_{i}}{2^{s_{i}}} \cdot \frac{c_{i}}{2} s_{i} 2^{s_{i}}=\frac{c}{2^{i+1}} c_{i} x_{i} s_{i}$. Thus $c_{i+1} x_{i+1} s_{i+1}=\left(c_{1} x_{1} s_{1}\right) \cdot \frac{c}{2^{3}} \cdot \frac{c}{2^{4}} \cdot \ldots \cdot \frac{c}{2^{i+1}}=c n \cdot \frac{c^{i-1}}{2^{(i+1) i / 2}} \geq\left(c^{\prime}\right)^{i^{2}} n$ for a constant $c^{\prime}$. So, for $i=\Theta\left(\log ^{*} n\right)$, there are at least $\left(c^{\prime}\right)^{\Theta\left(\left(\log ^{*} n\right)^{2}\right)} n=\Omega\left(\frac{n}{\log \log n}\right)$ stations that participate in phase $i$, for every $n$ large enough. It means that the algorithm initializes $\Omega\left(\frac{n}{\log \log n}\right)$ stations, i.e. labels them uniquely with $1,2, \ldots$.

By Lemma ??, at least a linear fraction of stations are assigned in Phase 1 unique ID's from the set $1, \ldots, 3 n$. In Phase 2 we run the algorithm just described and we get a set of stations $I$ labeled uniquely by numbers $1,2, \ldots,|I|$ such that $|I|=\Omega\left(\frac{n}{\log \log n}\right)$.

Phase 3 Paper [?] presents an algorithm for the strong RN that initializes $n$ stations in time $O(n)$ with energy $\operatorname{cost} O(\log \log n)$ and probability at least $1-1 / n$. It can be easily applied to the case when the approximation of the number of stations up to a constant multiplicative factor is given. Let $I$ be the set of stations that obtained consecutive ID's during the renumeration, $|I|=\Omega\left(\frac{n}{\log \log n}\right)$ with high probability. In Phase 3 we simulate the initialization algorithm from [?] using partial initialization trick (the elements of $I$ with ID's $1, \ldots, \min (|I|, n / 2)$ play a role of the first group and all other elements belong to the second group).

Recall that the initialization algorithm [?] works in time $O(n)$ and has energy cost $O(\log \log n)$. So one can easily check that our algorithm for the weak model works in time $O(n)$ and energy $O(\log \log n)$, too.

\section{General Simulation}

An algorithm for the strong RN can be simulated on a weak RN as follows: first we run initialization procedure as described in Section ??. Then we run the algorithm based on 
the partial initialization trick from Section ??: the stations $1, \ldots, n / 2$ are responsible for simulating the stations of the strong $\mathrm{RN}$, station $i$ simulating stations $2 i-1$ and $2 i$, the stations $n / 2+1, \ldots, n$ are used as the first group for the trick described in Section ??. Simulation of a step $j$ of the strong RN looks as follows: a station simulating stations $2 i-1$ and $2 i$ of the strong RN sends the bit 0 when both these stations send, does not send anything if neither $2 i-1$ nor $2 i$ send and send the bit 1 followed by the message sent by the station $2 i$ or $2 i-1$ otherwise. Station $n / 2+j$ listens at this step. If it receives the bit 1 followed by a message then it sends the message received at the next step, and these stations among $1, \ldots, n / 2$ listen, which represent the awake stations of the strong RN.

\section{References}

1. Y. Azar, A.Z. Broder, A.R. Karlin, U. Upfal, Balanced Allocations, SIAM Journal on Computing : 180-200 (1999).

2. J.L. Bordim, J. Cui, T. Hayashi, K. Nakano, S. Olariu, Energy efficient initialization protocols for ad-hoc radio networks, ISAAC'99, LNCS 1741, Springer-Verlag, 215-224.

3. W.C. Fifer, F.J. Bruno, Low Cost Packet Radio, Proc. of the IEEE, 75 (1987), 33-42.

4. L. Gąsieniec, A. Pelc, D. Peleg, The wakeup problem in synchronous broadcast systems, ACM PODC'2000, 113-121.

5. T. Jurdziński, M. Kutyłowski, J. Zatopiański, Energy-Efficient Size Approximation for Radio Networks with no Collision Detection, Computing and Combinatorics, COCOON'2002.

6. T. Jurdziński, M. Kutyłowski, J. Zatopiański, Efficient Algorithms for Leader Election in Radio Networks, ACM PODC'2002.

7. Jurdziński, T., Kutyłowski, M., Zatopiański, J.: Weak Communication in Radio Networks, Tech. Rep. CSR-02-04, Techn. Universität Chemnitz, Fakultät für Informatik, 2002.

8. E. Kushilevitz, Y. Mansour, Computation in Noisy Radio Networks, ACM-SIAM SODA '98, 236-243.

9. K. Nakano, S. Olariu, Randomized $O(\log \log n)$-round leader election protocols in radio networks, ISAAC'98, LNCS 1533, Springer-Verlag, 209-218.

10. K. Nakano, S. Olariu, Randomized leader election protocols for ad-hoc networks, SIROCCO'2000, Carleton Scientific, 253-267.

11. K. Nakano, S. Olariu, Randomized Leader Election Protocols in Radio Networks with No Collision Detection. ISAAC'2000, LNCS 1969, Springer-Verlag, 362-373.

12. K. Nakano, S. Olariu, Energy Efficient Initialization Protocols for Radio Networks with no Collision Detection, ICPP'2000, IEEE, 263-270.

13. R. Motwani, P. Raghavan, Randomized Algorithms, Cambridge University Press, 1995.

14. S. Rajasekaran, J. H. Reif, J. D. P. Rolim, (Eds.), Handbook on Randomized Computing, Kluwer Academic Publishers, 2001.

15. D.E. Willard, Log-logarithmic selection resolution protocols in multiple access channel, SIAM Journal on Computing 15 (1986) , 468-477.

16. IEEE Standard for Information Technology - LAN/MAN: Wireless LAN Medium Access Control (MAC), http://grouperieee.org/groups/802/11/main.html. 